\title{
Performance Modeling of Intelligent Car Parking Systems
}

\author{
Károly Farkas ${ }^{1,3}$, Gábor Horváth ${ }^{1,2}$, András Mészáros ${ }^{1,3}$, and Miklós Telek ${ }^{1,2}$ \\ 1 Budapest University of Technology and Economics \\ ${ }^{2}$ MTA-BME Information Systems Research Group \\ 3 Inter-University Center of Telecommunications and Informatics Debrecen
}

\begin{abstract}
The performance analysis of the car parking process in a parking lot with various levels of assistance is considered in the paper. The input of the model is the description of the parking lot and a Markovian description for the driver behavior, the set of computable performance measures contains the average time necessary for the user to reach the desired destination, the amount of cars moving in the parking lot at the same time (thus, the environmental strain), etc. To overcome the state space expansion, that makes the direct analysis of the model computationally infeasible, we apply a mean field limit based approximation whose accuracy is investigated with discrete event simulation.
\end{abstract}

\section{Introduction}

Since its introduction at the beginning of the century [2], the smart city concept enjoys an enormous attention from the research and development community, e.g., real-time traffic monitoring based car routing (i.e., Personal Navigation Assistant (PNA) devices with Traffic Message Channel (TMC) support) are commonly used in several countries, as well as the real-time tracking of Global Positioning System (GPS) equipped public transport devices. Intelligent parking systems, which we investigate in this paper belong to this category as well. In an intelligent car parking system the driver selects its destination on a smartphone application when entering the parking garage, and the parking system selects the optimal parking field and provides navigation aid to it, considering the occupancy situation.

Several such systems have been introduced in the literature in the recent years, e.g. [3], [1] or [5]. All these papers are focusing on the technical and implementation aspects of the problem, while in this paper, we focus on the stochastic performance analysis of such a parking system and calculate performance measures like the mean time spent by searching for a parking place and by walking to the destination, and the mean number of cars rambling around to find a place. The results can be used to quantify the benefits of an intelligent parking system.

The direct analysis of the overall parking system including the state of each individual car is inhibitory complex and that is why we propose a mean field limit based efficient approximate analysis [4]. 
The rest of the paper is organized as follows. Section 2 introduces the considered model. Section 3 provides the description of the behavior of the drivers, both in the un-assisted and in the assisted cases. The mean field model is defined in Section 4, and some numerical examples are investigated in Section 5.

\section{Model Description}

\subsection{The Floor Plan}

The architectural plan of the studied parking lot (Figure 1) contains a grid divisions which we use for representing the position of the cars in the garage by a discrete variable. These rectangular fields are identified by their row and column position $p=(r, c)$, and are held by set $\mathcal{P}=\{p\}$.

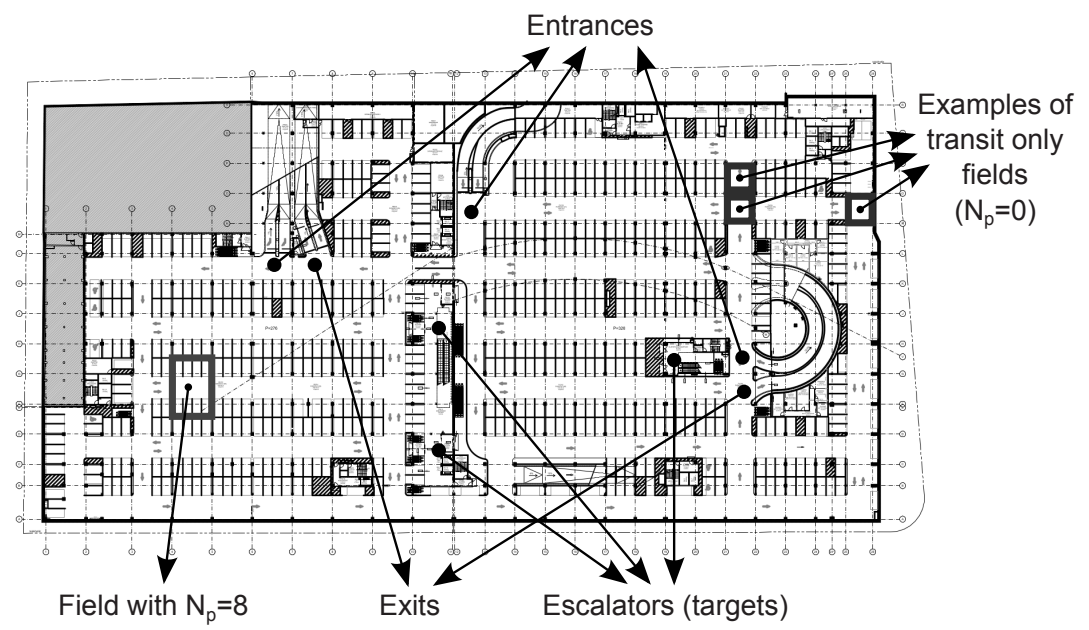

Fig. 1. Floor plan of the parking garage of the Allee mall in Budapest

Some fields contain a number of parking places, while others are serving as transit only. The maximal number of cars that can park at position $p$ is denoted by $N_{p}$, from which the free ones are denoted by $K_{p}$. The "transit only" and the "transit+parking" type fields are distinguished by $N_{p}=0$ and $N_{p}>0$ respectively. Additionally, there are fields with special purposes (see Figure 1).

- Set $\mathcal{E}$ contains the positions of the entrances of the garage.

- Set $\mathcal{T}$ contains the positions of the possible targets of the drivers.

- Set $\mathcal{X}$ contains the exit positions (to leave the garage).

The roads in the garage can be either one- or two-ways. The set of neighboring positions where a car can proceed after leaving $p$ is denoted by $\mathcal{N}(p)$, from which $\operatorname{succ}(p)$ represents the one that can be reached without changing direction. 
The shortest path between two fields will be used frequently in the sequel. When a tagged car is located at field $a \in \mathcal{P}$ we denote the next field along the shortest path towards field $b$ by $\vec{d}_{a, b}$ if the driving directions on the roads are respected, and by $d_{a, b}$, if they are not respected. The corresponding distances along the shortest path are denoted by $\vec{c}_{a, b}$ and $c_{a, b}$, respectively, where $\vec{d}_{a, b}$ and $\vec{c}_{a, b}$ applies to cars, while $d_{a, b}$ and $c_{a, b}$ applies to pedestrians.

\subsection{Behavior of the Cars}

The life cycle of a car consists of the following four phases.

1. It enters the garage at one of the entrances;

2. it searches for a free parking field, preferably as close to the target destination as possible;

3. it selects a free parking field and parks. The driver leaves the car behind proceeds the trip on foot.

4. Finally, after some time, the driver returns to the car and leaves the garage.

We assume that the selection of the entrance and the target is done by a random choice. The behavior of the drivers can be very different depending on their experiences. how much information the drivers have on the floor plan of the parking garage. Completely uninformed drivers have no information on the floor plan, and no clue on their target destination, whereas returning drivers know the floor plan and are able to take the distance information into consideration. In case of intelligent parking systems the occupancy information of the parking fields is available, too. The measurement based car behavior description is not available yet, thus our motion models are based on intuition. We are using the following four different motion models.

\section{- Uninformed drivers.}

In this case the motion of the cars is similar to a random walk. The actions the driver can take are:

- Stop and park. The more free places are in the current position, the easier is to park the car, thus the higher is the probability of parking.

- Change direction. The probability of choosing a direction is proportional with the number of parking places available in that direction.

- Go forward, without changing direction. It is not typical that a car changes direction at every possible places, thus this action has a higher probability than changing direction.

- Returning (distance aware) drivers.

If the driver has prior knowledge on the parking garage, apart of the available positions he/she can utilize the distance from the selected target destination. Thus, the probabilities associated with the three actions above will decrease with the distance from the destination.

If the utilization of the garage is high and parking fields close to the target are all occupied, distance aware drivers have a hard time to find an appropriate 
place to park. To avoid unrealistically long search phase, we introduce the patience of the drivers, which is a random variable. Once a driver becomes impatient, it gives up the distance preference and acts like an uninformed driver.

- Intelligent parking assist systems.

If an intelligent assist system is installed, the number and the distribution of the free parking places can be used in proposed guidance. When entering the garage, the driver selects the desired destination and the system directions to the best possible parking field. Due to the dynamic evolution of the occupancy of parking fields the parking assist system must re-calculate the optimal parking field and the corresponding directions continuously. We are considering two possible assist strategies:

- solely based on the distance from the desired destination, to minimize the walking distance,

- based on the distance from the desired destination and the driving distance, to minimize the driving plus walking time.

Regarding the departure phase of the life-cycle of a car, we assume that informative traffic signs guide the cars towards the exits on the shortest path.

\section{Formalizing the Car Motion Models}

The entrance, target and exit selections are assumed to be random choice with the following distributions.

$-q_{i}^{(E)}$ is the probability that the car enters at entrance $i \in \mathcal{E}$,

$-q_{j}^{(T)}$ is the probability that the driver intends to visit target $j \in \mathcal{T}$,

$-q_{k}^{(X)}$ is the probability that the driver chooses exit $k \in \mathcal{X}$ to leave.

\subsection{Parking Preference Functions}

We introduce two functions to characterize the parking preference of the drivers. Function $f_{p}^{(F)}$ represents the parking preference based on $K_{p}$, the number of free parking fields at position $p$. Obviously, if $K_{p}=0$ (all fields are occupied at $p$ ) we have that $f_{p}^{(F)}=0$, and $f_{p}^{(F)}$ is monotonously increasing with $K_{p}$, reflecting the observation that drivers prefer to park at positions where more free fields are available. If $f_{p}^{(F)}=1$, position $p$ is ideal for parking and every driver wants to take it. This function is approximated by a complementary Gaussian curve according to

$$
f_{p}^{(F)}=1-e^{-K_{p}^{2} / \sigma_{F}^{2}}
$$

where the variance parameter $\sigma_{F}^{2}$ will be used to tune the shape of this function. The choice of Gaussian curve is motivated by its frequent appearance in 

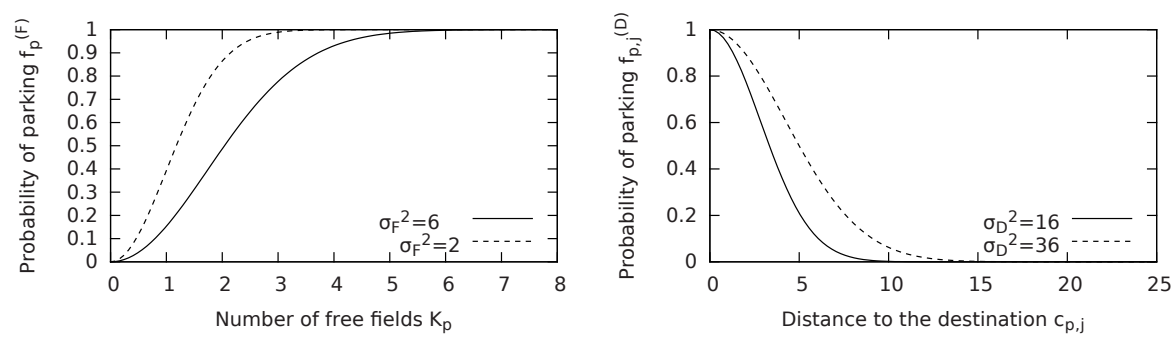

Fig. 2. Parking preference functions

stochastic models and its simplicity. We do not have experimental data (at all) to validate this choice.

Function $f_{p, j}^{(D)}$ represents the parking preference at position $p$ based on the distance of $p$ from the desired destination $j$. If the distance is $0(p=j)$, we have that $f_{p, j}^{(D)}=1$. Furthermore, $f_{p, j}^{(D)}$ decreases monotonously to zero with the distance $c_{p, j}$, since every driver wants to find a place as close to its destination as possible. Function $f_{p, j}^{(D)}$ is approximated by a Gaussian function (with the same lack of experimental data)

$$
f_{p, j}^{(D)}=e^{-c_{p, j}^{2} / \sigma_{D}^{2}}
$$

where the variance parameter $\sigma_{D}^{2}$ controls how important the distance is to the drivers. The shapes of the functions with the parameters used in the upcoming numerical example are depicted in Figure 2.

\subsection{Motion Model for Uninformed Drivers}

Our model for the uninformed driver is based on intuition, and might seem to be a bit artificial. We hope to improve it in the future based on empirical measurements. The reason to include it in this paper is to have a less sophisticated driver behavior in the comparison with the assisted case, for which we have an accurate model.

In our simple model, an uninformed driver can take one of the following actions while being at position $p \in \mathcal{P}$ :

- It can park with probability $q_{p}$, where $q_{p}=f_{p}^{(F)}$. The $\sigma_{F}^{2}$ parameter (corresponding to $f_{p}^{(F)}$, see (1)) depends on the average occupancy of the parking fields at position $p$, which the driver can estimate by looking around. The higher the occupancy of the parking lot is, the smaller $\sigma_{F}^{2}$ is, thus it gets more likely that the driver takes every single empty parking place it encounters. $\sigma_{F}^{2} \rightarrow 0$ corresponds to the case where the driver selects the first free place it encounters. With this setting the garage is filled up along concentric circles around the entrances. We use $\sigma_{F}^{2}>>0$, thus uninformed drivers just drive for a while to choose a place. 
- With probability $r_{p, i}, i \neq \operatorname{succ}(p)$ the driver decides to move on and change direction to a neighboring position $i \in \mathcal{N}(p)$. Choosing a direction where no free field is visible (e.g., because that field is for traversal only) has a non-zero probability as well (denoted by $1-\gamma)$. Accordingly, the weight corresponding to $r_{p, i}$ is $r_{p, i}=\gamma f_{i}^{(F)}+1-\gamma$.

- With probability $r_{p, \operatorname{succ}(p)}$ the driver decides to move on to the next position without changing direction. Not changing direction is more probable than changing direction, thus the weight assigned to this case is larger, it is $W\left(\gamma f_{\operatorname{succ}(p)}^{(F)}+1-\gamma\right), W>1$.

Thus, the probability of moving from position $p$ to position $i \in \mathcal{N}(p)$ is

$$
r_{p, i}= \begin{cases}\frac{1}{\varphi_{1}}\left(\gamma f_{i}^{(F)}+1-\gamma\right), & i \neq \operatorname{succ}(p), \\ \frac{1}{\varphi_{1}} W\left(\gamma f_{\operatorname{succ}(p)}^{(F)}+1-\gamma\right), & i=\operatorname{succ}(p) \\ 0 & \text { otherwise }\end{cases}
$$

where $\varphi_{1}=\sum_{j \in \mathcal{N}(p), j \neq s u c c(p)} \gamma f_{j}^{(F)}+1-\gamma+W\left(\gamma f_{\text {succ }(p)}^{(F)}+1-\gamma\right)$. Hereafter we assume that a car does not turn over $180^{\circ}$ and go back to its preceding position unless this is the only possibility to proceed.

\subsection{Motion Model for Distance Aware Drivers}

In case of returning drivers the distance information is also available when making the parking decision. The probability that the driver stops and parks at position $p$ depends both on the number of free parking fields $K_{p}$ and on the distance to the destination $j$ as $q_{p}=f_{p}^{(F)} \cdot f_{p, j}^{(D)}$. The variance parameters $\sigma_{F}^{2}$ and $\sigma_{D}^{2}$ of distance aware drivers are less than the ones in the uninformed case, as returning drivers are more determined.

If the driver decides not to park, the distance information plays a role in the distribution of the next position of the car, too, according to

$$
r_{p, i}= \begin{cases}\frac{1}{\varphi_{2}}\left(\gamma f_{i}^{(F)}+1-\gamma\right) f_{i, j}^{(D)}, & i \neq \operatorname{succ}(p), \\ \frac{1}{\varphi_{2}} W\left(\gamma f_{i}^{(F)}+1-\gamma\right) f_{\operatorname{succ}(p), j}^{(D)}, & i=\operatorname{succ}(p), \\ 0 & \text { otherwise }\end{cases}
$$

where $\varphi_{2}=\sum_{j \in \mathcal{N}(p), m \neq \operatorname{succ}(p)}\left(\gamma f_{m}^{(F)}+1-\gamma\right) f_{m, j}^{(D)}+W\left(\gamma f_{\operatorname{succ}(p)}^{(F)}+1-\gamma\right) f_{\text {succ }(p), j}^{(D)}$.

To avoid endless rambling the patience of the drivers has to be included in the model as well. We assume that the patience follows a discrete phase-type (DPH) distribution defined by initial probability vector $\alpha$ and transient transition probability matrix $\boldsymbol{A}$, yielding $P($ patience $=k)=\alpha \boldsymbol{A}^{k-1}(\boldsymbol{I}-\boldsymbol{A}) \mathbb{1}$. Once the patience of the driver is over, it gives up optimizing on the distance and switches to the uninformed strategy defined in Section 3.2. 


\subsection{Motion Model in the Presence of Intelligent Parking Systems}

The intelligent parking system continuously sends the suggested driving direction towards the most appealing parking field to the driver. Given that the car is currently at position $p$ and the desired destination is target $j$, the appeal of a field at position $i$ (denoted by $a_{p, j, i}$ ) is derived from the parking preference function $f_{i}^{(F)}$ and $f_{p, j, i}^{(D)^{\prime}}$, which depends on the distance to be driven from $p$ to $i$ and the walking distance from $i$ to the destination $j$, that is $f_{p, j, i}^{(D)^{\prime}}=e^{-\left(\xi_{d} \vec{c}_{p, i}+\xi_{w} c_{i, j}\right)^{2} / \sigma_{D}^{2}}$, where $\xi_{d}$ and $\xi_{w}$ determine the weights of driving and walking distance in the decision, respectively. If the parking system optimizes on solely the walking distance, we have that $\xi_{d}=0$ and $\xi_{w}=1$.

The appeal $a_{p, j, i}$ is then modeled by $a_{p, j, i}=f_{i}^{(F)} \cdot f_{p, j, i}^{(D)^{\prime}}$, and the best position $p^{\text {opt }}$ is selected according to $p^{o p t}=\arg \min _{i \in \mathcal{P}} a_{p, j, i}$. We also take into account that there are drivers that do not follow the guidance of the parking system till the end, but with probability $U \cdot f_{p}^{(F)} \cdot f_{p, j}^{(D)}$ can park to a parking field even if it is not the recommended one (parameter $U$ stands for drivers independence). All in all, an assisted car at position $p$ heading to target $j$ can take the following actions.

- A car stops and park with probability

$$
q_{p}= \begin{cases}1, & \text { if } p=p^{o p t} \\ U \cdot f_{p}^{(F)} \cdot f_{p, j}^{(D)}, & \text { otherwise }\end{cases}
$$

- Given that the car did not park, it moves forward to position $i$ with probability

$$
r_{p, i}= \begin{cases}1, & \text { if } i=\vec{d}_{p, p^{o p t}} \\ 0, & \text { otherwise }\end{cases}
$$

\subsection{Distribution of the Parking Time}

The duration of parking is modeled by a discrete phase type distribution with initial probability vector and transient transition probability matrix denoted by $\beta$ and $\boldsymbol{B}$, respectively, that is $P$ (parking time $=k)=\beta \boldsymbol{B}^{k-1}(\boldsymbol{I}-\boldsymbol{B}) \mathbb{1}$.

After parking the cars leave the garage along the shortest path to the selected exit. In the considered closed system scenario when a car leaves the garage, a new car enters immediately to keep the number of cars in the garage constant. We apply the closed system scenario to eliminate the randomness caused by the randomly changing load (number of cars) and to amplify the performance implications of the considered driver behavior. 


\section{The Mean-Field Model}

We introduce a discrete time model in this section to characterize the state of the parking garage. Each car being in search or leaving phase moves exactly one field along the grid in each time step.

\subsection{The State Space of the System}

Cars in the garage are either search for a parking field, or parked, or leaving. For cars in the search phase we need to keep track of

- the current position $p$,

- the desired target destination $j$,

- the current orientation of the car o (to avoid complete turn-overs),

- and, in case of the distance-aware strategy,

- the phase of the DPH distribution representing the patience $n$.

- a flag $f$ indicating that the car lost patience and gave up optimizing on distance

For parked cars we have to follow the

- the position of the car $p$,

- the phase of the DPH corresponding to the parking time $m$.

Finally, for leaving cars we have to include into the state space

- the current position of the car $p$,

- and the selected exit where the car is heading to $x$.

Thus, the state of a car at time $k$ can be represented by

$$
\mathcal{S}_{k} \in\{(\text { search }, p, j, o, n, f)\} \cup\{(\text { parked }, p, m)\} \cup\{(\text { leaving }, p, x)\} .
$$

if the cars are using a distance aware strategy, otherwise it can be represented by

$$
\mathcal{S}_{k} \in\{(\text { search }, p, j, o)\} \cup\{(\text { parked }, p, m)\} \cup\{(\text { leaving }, p, x)\} .
$$

\subsection{State Transitions}

The possible state transitions of cars being in the search phase at time $n$ are the following in case of the uninformed and the assisted case.

$$
\begin{aligned}
P\left(\mathcal{S}_{k+1}=(\text { parked }, p, m) \mid \mathcal{S}_{k}\right. & =(\text { search }, p, j, o, n, f))=q_{p_{e f f}} \beta_{m}, \\
P\left(\mathcal{S}_{k+1}=\left(\text { search }, i, j, o^{\prime}, n, f\right) \mid \mathcal{S}_{k}\right. & =(\text { search }, p, j, o, n, f))=r_{p, i}
\end{aligned}
$$

where $q_{p}$ and $r_{p, i}$ depends on the car motion model (see Section 3), and $o^{\prime}$ is the orientation of the car after moving from $p$ to $i$. Note that instead of the parking probability $q_{p}$, a modified quantity, the effective parking probability $q_{p_{e f f}}$ is 
used in the formula to ensure that the parking capacity $N_{p}$ of field $p$ is never exceeded (in the mean sense). If there are $S_{p}$ cars on the same position, and each individual car wants to park with probability $q_{p}$, the mean number of parking cars in the next step would be $S_{p} q_{p}$, that can be larger than the capacity of $p$. Therefore the effective parking probability is given by

$$
q_{p_{e f f}}=\left\{\begin{array}{ll}
q_{p}, & \text { if } S_{p} q_{p} \leq N_{p}, \\
q_{p} K_{p} / N_{p} & \text { otherwise }
\end{array}= \begin{cases}q_{p}, & \text { if } s_{p} q_{p} \leq n_{p} \\
q_{p} k_{p} / n_{p} & \text { otherwise }\end{cases}\right.
$$

where $S_{p}$ denotes the number of cars being in the search phase at position $p$ and $s_{p}=S_{p} / N, n_{p}=N_{p} / N, k_{p}=K_{p} / N$ are the population normalized parameters which equally define the transition probability.

If the cars are moving according to the distance aware strategy, the patience information needs to be taken into account as well, hence we have

$$
\begin{aligned}
& P\left(\mathcal{S}_{k+1}=(\text { parked }, p, m) \mid \mathcal{S}_{k}=(\text { search }, p, j, o, n, \text { false })\right)=q_{p_{e f f}}^{(D A)} \beta_{m}, \\
& P\left(\mathcal{S}_{k+1}=(\text { parked }, p, m) \mid \mathcal{S}_{k}=(\text { search }, p, j, o, n, \text { true })\right)=q_{p e f f}^{(U I)} \beta_{m}, \\
& P\left(\mathcal{S}_{k+1}=\left(\text { search }, i, j, o^{\prime}, n, \text { true }\right) \mid \mathcal{S}_{k}=(\text { search }, p, j, o, n, \text { true })\right)=r_{p, i}^{(U I)}, \\
& P\left(\mathcal{S}_{k+1}=\left(\text { search }, i, j, o^{\prime}, n^{\prime}, \text { false }\right) \mid \mathcal{S}_{k}=(\text { search }, p, j, o, n, \text { false })\right)=r_{p, i}^{(D A)} \boldsymbol{A}_{n, n^{\prime}}, \\
& P\left(\mathcal{S}_{k+1}=\left(\text { search }, i, j, o^{\prime}, n, \text { true }\right) \mid \mathcal{S}_{k}=(\text { search }, p, j, o, n, \text { false })\right) \\
& \quad=r_{p, i}^{(D A)}\left(1-\sum_{n^{\prime}} \boldsymbol{A}_{n, n^{\prime}}\right)
\end{aligned}
$$

where $q_{p_{\text {eff }}}^{(U I)}$ and $q_{p_{e f f}}^{(D A)}$ are the effective parking probabilities associated with the uninformed and distance aware case, respectively, and the routing probabilities $r_{p, i}^{(U I)}$ and $r_{p, i}^{(D A)}$ are distinguished in the same way.

Cars being in the parked phase can either stay parked or enter to the leaving state according to

$$
\begin{aligned}
& P\left(\mathcal{S}_{k+1}=\left(\text { parked }, p, m^{\prime}\right) \mid \mathcal{S}_{k}=(\text { parked }, p, m)\right)=\boldsymbol{B}_{m, m^{\prime}} \\
& P\left(\mathcal{S}_{k+1}=(\text { leaving }, p, x) \mid \mathcal{S}_{k}=(\text { parked }, p, m)\right)=\left(1-\sum_{m^{\prime}} \boldsymbol{B}_{m, m^{\prime}}\right) \cdot q_{x}^{(X)} .
\end{aligned}
$$

Finally, the state transition probabilities corresponding to leaving cars are

$$
\begin{aligned}
& P\left(\mathcal{S}_{k+1}=\left(\text { leaving }, \vec{d}_{p, x}, x\right) \mid \mathcal{S}_{k}=(\text { leaving }, p, x)\right)=1, \quad \text { if } p \neq x, \\
& P\left(\mathcal{S}_{k+1}=\left(\text { search }, e, j, o_{e}, n, \text { false }\right) \mid \mathcal{S}_{k}=(\text { leaving }, p, x)\right)=q_{e}^{(E)} q_{j}^{(T)} \alpha_{n} \text {, if } p=x \text {, }
\end{aligned}
$$

given that the orientation of the cars entering at entrance $e$ is $o_{e}$.

\subsection{The Occupancy Vector and Its Mean Field Limit}

Let $X_{n}^{N}(k), n=1, \ldots, N$ denote the state of car $n$ at time step $k$, where $N$ is the (constant and finite) number of cars in the garage. The state transition 
probabilities given in Section 4.2 define a discrete time Markov chain (DTMC) for a given number of cars, where the DTMC keeps track of the states of all the cars in the garage. In this Markov chain the stochastic behavior of cars are interdependent because the movement (the state transition probabilities) of a given car depends on the positions of the other cars, but it is important to note that the state transition probabilities do not depend on the position of particular cars $X_{n}^{N}(k)$, but only on the number of cars which stay in the grids (i.e., vector $M^{N}$ defined below). We refer to this property as density dependence.

When $N$ is large ( $\geq$ several tens), which is the common case in practice, the state space of the DTMC gets extremely large and the analysis of this large DTMC becomes infeasible. To overcome this limitations we can approximate the DTMC which describe the behavior of $N$ (finite) cars in the parking lot with its mean field limit, which is obtained when the number of cars increases to infinity, due to the density dependence of the model.

In order to evaluate the mean field limit we introduce the occupancy measure ([4]) which is a row vector $M^{N}(k)=\left[M_{i}^{N}(k), i \in \mathcal{S}\right]$ where $M_{i}^{N}(k)$ is the proportion of cars being in state $i$ at time step $k$ which is $M_{i}^{N}(k)=\frac{1}{N} \sum_{n=1}^{N} I_{\left\{X_{n}^{N}(k)=i\right\}}$, with $I_{\{\}}$being the indicator function, and introduce the normalized versions of the transition probability functions.

The state transitions probabilities given in Section 4.2 depend on the number of cars which stay in the grids. E.g., $q_{p_{\text {eff }}}$ depends on $K_{p}$ which is the number of free parking fields at position $p$, and on $S_{p}$ which is the number of cars searching a parking field at $p$. The population normalized versions of these quantities can be expressed from the occupancy vector as $k_{p}(k)=n_{p}-\sum_{i \in\{(\text { parked,p,m })\}} M_{i}^{N}(k)$, $s_{p}(k)=\sum_{i \in\{(\operatorname{search}, p, j, o, n, f)\}} M_{i}^{N}(k)$. Let $\bar{\Pi}\left(M^{N}(k)\right)$ denote the state transition probability matrix containing the state transitions probabilities given in Section 4.2 as a function of the population normalized number of cars in different states. The mean field limit of the occupancy vector $M^{N}(k)$, denoted by $M(k)$, satisfies the difference equation $M(k+1)=M(k) \bar{\Pi}(M(k))$ [4]. This difference equation is much less expensive to compute than the direct analysis of the large DTMC model representing the product space of the states of all cars in the garage.

The mean field limit is obtained when the number of cars and the capacity of the parking lot increases proportionally (i.e., the "size" of the cars decreases to zero). However, the mean field model gives an approximate analysis of the system, since the number of cars being in state $i$ calculated by $N \cdot M_{i}(k)$ is a real number, while it is an integer variable in the reality.

\subsection{Performance Measures}

We evaluate the following performance measures based on the occupancy measure $M(k)$, the mean driving time to parking, $L_{S}$, the mean walking distance from the selected parking field to the target destination, $L_{W}$, the mean of the total latency including the driving and walking time. $L_{T}$, and the ratio of cars moving in the garage at the same time (either in search or leaving phase), $C$. At time $k C$ is obtained as $C(k)=\sum_{i \in\{(\operatorname{search}, p, j, o, n, f)\} \cup\{(\text { leaving, }, x)\}} M_{i}(k)$. 
To compute the rest of the performance measures we introduce $\ell_{j, p}(t)$, which is the probability that a car heading to target $j$ finds a parking field at time $t$ and position $p$. For $\ell_{j, p}(t)$ we have $\ell_{j, p}(t)=a \cdot\left(\bar{\Pi}_{\text {search, search }}\right)^{t-1} \cdot b_{j, p}$, where row vector $a$ reflects the starting state of an individual car. The entries of $a$ are given by

$$
a_{i}= \begin{cases}q_{e}^{(E)} q_{j}^{(T)}, & \text { if } i=\left(\text { search }, e, j, o_{e}, n, f\right), \\ 0, & \text { otherwise. }\end{cases}
$$

Furthermore, $\bar{\Pi}_{\text {search, search }}$ is derived from $\lim _{k \rightarrow \infty} \bar{\Pi}(M(k))$ by setting entries but the ones belonging to the search phases to zero. Thus, the entries of $\left(\bar{\Pi}_{\text {search,search }}\right)^{t}$ are the probabilities that the car is still in the search phase after time $t$ with the corresponding state transitions. Finally, $b_{j, p}$ is a column vector whose entry $i$ is the probability that a car in a search phase heading to target $j$ being at position $p$ stops and selects a parking field. It can be obtained as

$$
\left(b_{j, p}\right)_{i}= \begin{cases}\sum_{\forall m} P\left(\mathcal{S}_{k+1}=(\text { parked }, p, m) \mid \mathcal{S}_{k}=i\right), & \text { if } i=(\text { search }, p, j, o, n, f), \\ 0, & \text { otherwise. }\end{cases}
$$

With $\ell_{j, p}(t)$ the mean search time is $L_{S}=\sum_{t=0}^{\infty} t \cdot \sum_{\forall j \in \mathcal{T}, p \in \mathcal{P}} \ell_{j, p}(t)$, and the mean walking distance to the target is $L_{W}=\sum_{\forall j \in \mathcal{T}, p \in \mathcal{P}} c_{p, j} \sum_{t=0}^{\infty} \ell_{j, p}(t)$.

To calculate $L_{T}$ we have to take into consideration that walking is slower than driving. By denoting the time required to walk through a field in the grid relative to the driving time by $R$ the total time to target is given by $L_{T}=$ $\sum_{\forall j \in \mathcal{T}, p \in \mathcal{P}} \sum_{t=0}^{\infty}\left(t+c_{p, j} R\right) \cdot \ell_{j, p}(t)$.

\section{$5 \quad$ Numerical Experiments}

We implemented the mean field method in $\mathrm{C}++$ environment and compared the performance of the parking strategies discussed in the paper ${ }^{1}$. The floor plan corresponds to the "Allee" shopping mall in Budapest. The parameters of the model have been determined by intuition due to the lack of real data according to Table 1. The DPH distribution generating the parking time has a mean of 4000 seconds and the squared coefficient of variation is 1.5. The corresponding parameters are

$$
\beta=\left[\begin{array}{lll}
0.16 & 0.84 & 0
\end{array}\right], \boldsymbol{B}=\left[\begin{array}{ccc}
0.99988 & 0.00012 & 0 \\
0 & 0.99925 & 0.00075 \\
0 & 0 & 0.99925
\end{array}\right]
$$

\footnotetext{
${ }^{1}$ The software is open source and can be downloaded from http://www.hit.bme.hu/ ghorvath/software
} 
Table 1. Values of the parameters used in the examples

\begin{tabular}{cc}
\hline Strategy & Parameters \\
\hline Uninformed & $\sigma_{F}^{2}=6 ; \gamma=0.3 ; W=3$ \\
\hline Distance aware & $\sigma_{F}^{2}=2 ; \gamma=0.2 ; W=1.5 ; \sigma_{D}^{2}=16 ;$ \\
& $\alpha=\left[\begin{array}{lll}0.321 & 0.379 & 0\end{array}\right] ; \boldsymbol{A}=\left[\begin{array}{ccc}0.99679 & 0.00321 & 0 \\
0 & 0.99 & 0.01 \\
0 & 0 & 0.99\end{array}\right]$ \\
\hline Assisted, case 1. & $\sigma_{F}^{2}=2 ; \sigma_{D}^{2}=36 ; U=0.05 ; \xi_{d}=0 ; \xi_{w}=1$ \\
\hline Assisted, case 2. & $\sigma_{F}^{2}=2 ; \sigma_{D}^{2}=36 ; U=0.05 ; \xi_{d}=0.2 ; \xi_{w}=0.8$ \\
\hline
\end{tabular}

\subsection{Distribution of the Occupied Parking Fields}

Figure 3 compares the mean occupancy of the parking fields under light load $(N=250)$. When the drivers are uninformed, they choose lightly occupied fields for parking along the main roads on the garage, instead of the ones located close to the targets.

In the distance aware case it is visible that the cars are parking around the three escalator entrances of the garage. Observe, however, that the garage can be divided into two main parts, and there is only a single possibility to move to the left part from the right one. Therefore there are drivers who choose sub-optimal parking field at the right part because they do not find the way to the less loaded left part. This situation is reflected on the heat map as well, the circular region corresponding to the more occupied parking fields is asymmetric, there are much more cars parking at the right side.

The occupancy of the parking fields is better distributed around the targets in the first assisted case (where only the walking distance is the subject of optimization), since the intelligent parking system is able to guide the cars to the left part if there are more free parking fields there.

The heat map belonging to the second assisted case (where the driving time and the walking time are both optimized) does not differ too much in this particular example.

The execution times were $2 \mathrm{~ms}, 8 \mathrm{~ms}, 2 \mathrm{~ms}, 21 \mathrm{~ms}$ per iteration in the uninformed, distance aware, the first and the second assisted case, respectively on an average $\mathrm{PC}$ with a $3.4 \mathrm{GHz} \mathrm{CPU}$ and $4 \mathrm{~GB}$ of memory.

\subsection{Comparing the Uninformed Model with Simulation}

To assess the precision of the mean field approximation we implemented the uninformed driver case in $\mathrm{OMNeT}++[6]$, which is a $\mathrm{C}++$ based framework for discrete event simulation. 

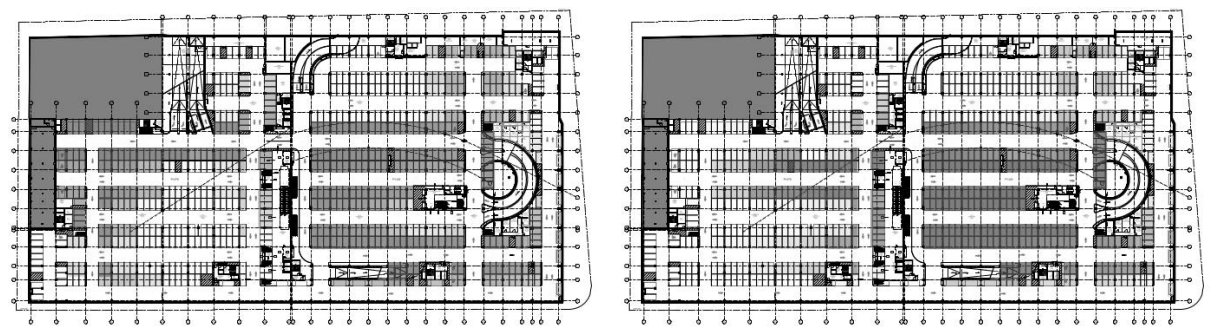

Uninformed drivers

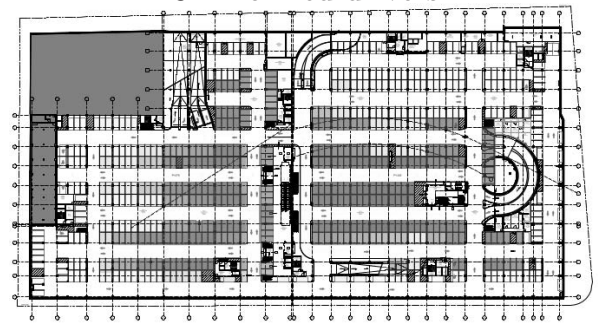

Distance aware drivers

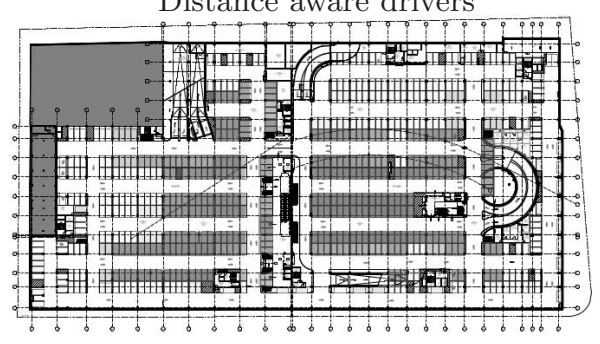

Assisted case 1.

Assisted case 2.

Fig. 3. The distribution of the occupied parking fields

We examined the mean occupancy of the parking fields as well as the average searching times and walking distances of the drivers. The simulations were run for a total of $10^{6}$ parking events, of which the first $10^{5}$ were considered a warmup period thus they were not taken into account in the statistics calculations. Between several test runs the difference in mean occupancy was less than 0.005 for more than $90 \%$ of the parking fields (with an average less than 0.002), while the maximum difference was around 0.02 . The relative differences between the searching and walking times were both less than $0.2 \%$ for every test run.

In the comparison we made the inspection for four different loads $(N=$ $100,300,400,500)$. To demonstrate the transition between the simulation and the mean field model we introduce the $\omega$ scaling parameter $(1 \leq \omega<\infty)$. The meaning of this parameter in the simulation is the following. If $\omega>1$, the number of cars in the system and the parking fields at each position are multiplied by $\omega$. Furthermore, to compensate the scaling $f_{p}^{(F)}$ becomes $f_{p, \omega}^{(F)}=1-e^{-K_{p}^{2} /\left(\omega \sigma_{F}\right)^{2}}$. For $\omega=1$ the model corresponds to the original physical realization. When $\omega \rightarrow \infty$ the behavior converges to the mean field model. Figure 4 shows the cumulative distribution functions of the errors of the mean occupancy for light and heavy loads. In accordance with the expectations the errors decrease when increasing $\omega$. It can also be seen, that the precision of the mean field model decreases with the increasing load. Table 2 shows the performance measures of interest. The same tendency can be observed in this case as well, however, the error is small except for the mean search time in the $N=500$ case, therefore the mean field approximation proved to be quite precise for low and medium load. 
Table 2. Performance measures of simulation and mean field model

\begin{tabular}{cccccc}
\cline { 3 - 6 } & & \multicolumn{3}{c}{ Number of cars in the garage } \\
\cline { 3 - 6 } Simulation & Mean search time & 7.57176 & 12.1356 & 16.411 & 37.8561 \\
\hline \multirow{2}{*}{ Mean field model } & Mean walking distance & 8.97576 & 10.3427 & 10.8522 & 10.7663 \\
\cline { 2 - 6 } & Mean search time & 7.61508 & 12.5713 & 16.9241 & 47.2762 \\
\cline { 2 - 6 } & Mean walking distance & 9.00815 & 10.5659 & 10.8671 & 10.948 \\
\hline
\end{tabular}
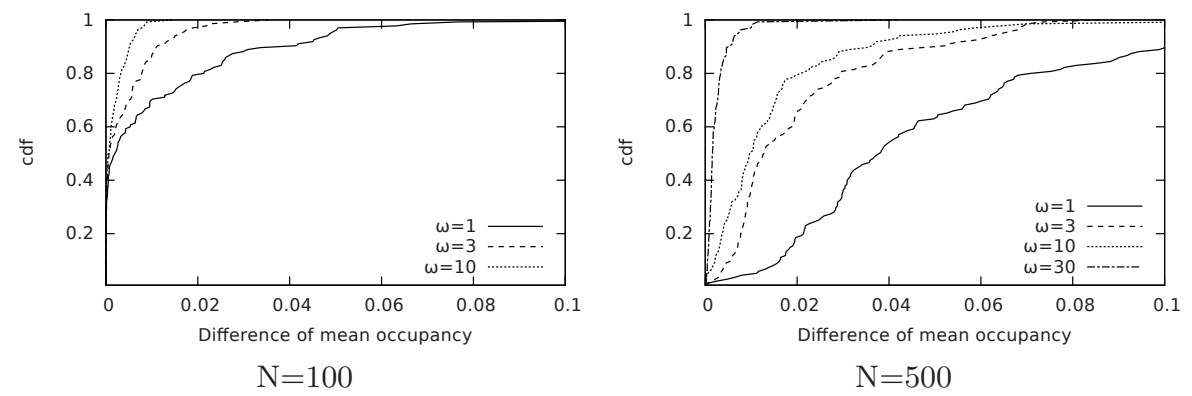

Fig. 4. The effect of scaling to the error of mean occupancy

\subsection{The Effect of the Load of the Garage}

Figure 5 depicts the performance measures as the function of the load. To record the results $5 \cdot 10^{5}$ iterations were executed, which was enough to get high precision results in the cases where the iteration converged. In the uninformed and the distance aware cases the convergence was fast and found a (supposedly) global at attractor from any random initial states. However, with the assisted strategies the mean field iteration did not converge, it had an oscillating behavior. The corresponding plots on the figures show the average of the results from 10 different random initial points and also have error bars indicating the minimum and maximum values obtained.

With the increase of the load, the performance of both the informed and distance aware strategies drop sharply. Cars spend too much time rambling around to find a position where the number of free spaces and the distance from the target is appropriate. To be fair, our model did not include an important factor: in the reality the behavior of the drivers (in particular the $\sigma_{F}$ and $\sigma_{D}$ parameters) depends on the utilization of the garage.

Nevertheless, the results make it clear that the intelligent parking systems can be really efficient. Both variants were able to reduce the time to reach the target, including the search phase and the walking time. Especially the "assisted case 2.", that takes the total time to target into account, was successful in reducing the number of moving cars in the garage even at high load, which is beneficial from the environmental protection point of view as well. 

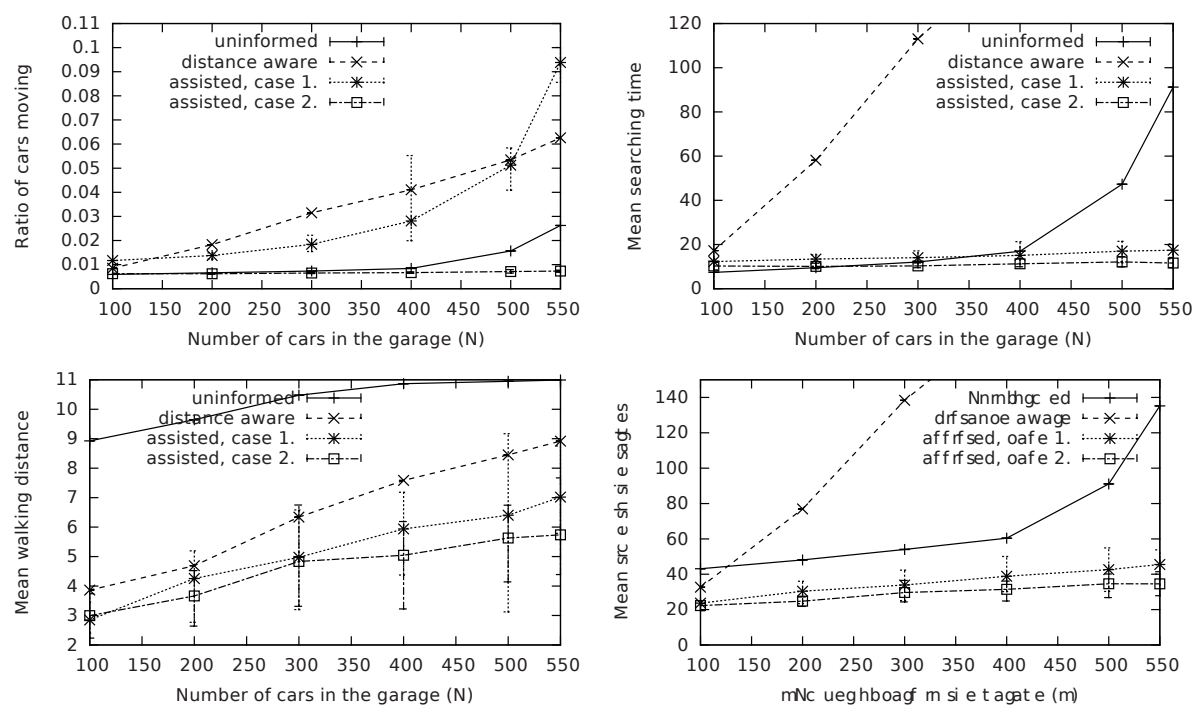

Fig. 5. The performance measures as the function of the load

Acknowledgment. This work was supported by the Hungarian research project OTKA K101150, by the European Union (co-financed by the European Social Fund) through the TAMOP-4.2.2C-11/1/KONV-2012-0001 project, and by the János Bolyai Research Scholarship of the Hungarian Academy of Sciences.

\section{References}

1. Bojja, J., Kirkko-Jaakkola, M., Collin, J., Takala, J.: Indoor 3D navigation and positioning of vehicles in multi-storey parking garages. In: Acoustics, Speech and Signal Processing (ICASSP), pp. 2548-2552. IEEE (2013)

2. Caragliu, A., Bo, C.D., Nijkamp, P.: Smart cities in Europe. Technical report (2009)

3. Gódor, G., Huszák, Á., Farkas, K.: Intelligent indoor parking. In: Proc. of Global Virtual Conference, GV-CONF (2013)

4. Le Boudec, J.-Y., McDonald, D., Mundinger, J.: A generic mean field convergence result for systems of interacting objects. In: Quantitative Evaluation of Systems, QEST, pp. 3-18. IEEE (2007)

5. Liu, J., Chen, R., Chen, Y., Pei, L., Chen, L.: iParking: An intelligent indoor location-based smartphone parking service. Sensors 12(11), 14612-14629 (2012)

6. Varga, A.: The OMNeT++ discrete event simulation system. In: Proceedings of the European Simulation Multiconference (ESM 2001), vol. 9, p. 185. sn (2001) 